\title{
Understanding the Origin and Evolution of Meteoritic Refractory Minerals Through Transmission Electron Microscopy
}

\author{
Tarunika Ramprasad $^{1 *}$, Pierre Haenecour ${ }^{2}$, Laura Seifert ${ }^{2}$ and Thomas J. Zega ${ }^{1,2}$ \\ 1. Department of Material Science and Engineering, University of Arizona, Tucson, USA. \\ 2. Lunar and Planetary Laboratory, University of Arizona, Tucson, USA. \\ * Corresponding author: tarunika@lpl.arizona.edu
}

Carbonaceous chondrite meteorites contain $\mathrm{mm}$ - to $\mathrm{cm}$-sized objects called calcium-aluminum-rich inclusions (CAIs) [1]. Based on isotopic age dating and thermodynamic predictions, their refractory mineral components are widely accepted to be the first formed solids in the solar protoplanetary disk [24]. CAIs are acknowledged to have formed initially via gas-phase condensation some 4.56 billion years ago, but many inclusions are known to have experienced subsequent thermal and aqueous processing in the solar nebula and on their parent asteroidal bodies [5]. The structure and chemistry of CAIs record the environments under which they formed and to which they were subsequently exposed. Analytical techniques with high spatial resolution are necessary to determine their structure and chemistry and gain insight into their formation and secondary processing. Thus, transmission electron microscopy can provide valuable information on CAI origins and insight into the earliest processes in our solar system.

Refractory assemblages were identified in a compact type-A (CTA) CAI from the Northwest Africa (NWA) 5028 CR2 chondrite. The CTA inclusion and the constituent mineral assemblages occur in a petrographic thin section of NWA 5028 (Center for Meteorite Studies, Arizona State University collection \#1845-5) and were chemically mapped using a Cameca SX-100 electron microprobe, located at the Lunar and Planetary Laboratory, University of Arizona (UA/LPL) [6,7]. The CAI has a core of melilite $\left(\mathrm{Ca}_{2} \mathrm{Al}_{2} \mathrm{SiO}_{7}\right)$, spinel, and perovskite, and is surrounded by a multi-mineralic rim. Assemblages of spinel $\left(\mathrm{MgAl}_{2} \mathrm{O}_{4}, 1397 \mathrm{~K}\right)$ and perovskite $\left(\mathrm{CaTiO}_{3}, 1593 \mathrm{~K}\right)$, within the inclusion, were selected for analysis in the TEM because they are believed to have condensed at high temperatures and are probes of nebular condensation. Their chemistry and crystal structure can therefore provide information on the thermodynamic conditions (temperature and pressure) prevalent in our protoplanetary disk.

The selected assemblages (two from the interior and one from the rim) were extracted and thinned to electron transparency using previously described methods [8] with an FEI Helios NanoLab 660 focusedion beam (FIB) scanning electron microscope (SEM) equipped with an EDAX energy-dispersive X-ray spectrometer (EDS), located at UA/LPL. The sections from the CAI were analyzed using a $200 \mathrm{keV}$ spherical-aberration corrected Hitachi HF5000 scanning transmission electron microscope (S/TEM) and a 30kV Hitachi SU9000 SEM/STEM, located at UA/LPL. The HF5000 and SU9000 are both equipped with Oxford Instruments (X-max) EDS detectors. The HF5000 has twin EDS detectors with a large solid angle $(2.0 \mathrm{sr})$ of collection. EDS and selected area electron diffraction (SAED) were used in conjunction for phase identification.

Section \#1 from the interior is composed of a $6-\mu \mathrm{m}$ wide spinel grain with a silicate-perovskite inclusion and a 5- $\mu \mathrm{m}$ wide perovskite grain [6,7]. Section \#2 from the rim is composed of three perovskite grains (one with a spinel inclusion) embedded in a large spinel grain, and contains veins and rims around the grains of material with compositions consistent with Fe-rich silicates [6,7]. The Fe-silicates have needlelike morphologies and occur across the width and length of the veins in crosslinked patterns. Section \#3 
from the interior is composed of two spinel grains $(3.5 \mu \mathrm{m}$ and $6.2 \mu \mathrm{m})$ and one perovskite grain $(2.7$ $\mu \mathrm{m})$ partially surrounded by a Ca-Al silicate phase (Fig. 1). The EDS maps also show the presence of some Cl-rich regions in the silicate phase (Fig. 1).

The perovskite inclusion within the spinel grain (\#1) is consistent with the spatial relationship predicted by equilibrium thermodynamics, i.e., sequential condensation of the mineral phases [2,3]. While the spinel inclusion within the perovskite grain (\#2) seems to contradict this predicted core-shell structure, it is consistent with the sequence derived from CAI melt-solidification experiments [9]. The Fe-silicates in the veins (\#2) are morphologically and compositionally consistent with Fe-rich phyllosilicates previously described to have formed via aqueous alteration [10]. Chlorine is a highly volatile element and its incorporation requires low-temperature processing. We therefore hypothesize that the $\mathrm{Cl}$-rich phases (\#3) were formed through low-temperature iron-alkali-halogen metasomatism such as those described by $[5,10]$. FIB-enabled TEM analysis shows that the microstructure of the CAI records a complex history that includes condensation, melt solidification, and aqueous processing [11].

\section{References:}

[1] GJ MacPherson in “Treatise on Geochemistry. Vol I: Meteorites, Comets and Planets”, ed. Davis A.M., (Elsevier, Waltham) p. 201.

[2] DS Ebel in "Meteorites and the Early Solar System II", ed. Lauretta D.S. and McSween Jr. H.Y., (University of Arizona Press, Tucson) p. 253.

[3] K Lodders, The Astrophysical Journal 591 (2003), p. 1220.

[4] Y Amelin, Science 297 (2002), p. 1678.

[5] AJ Brearley in "Treatise on Geochemistry. Vol I: Meteorites, Comets and Planets", ed. Davis A.M., (Elsevier, Waltham) p. 247.

[6] T Ramprasad et al., Lunar \& Planetary Sciences Conference (2018) Abstract \#2900.

[7] T Ramprasad et al., Microscopy \& Microanalysis Conference (2018).

[8] TJ Zega et al., Meteoritics \& Planet. Sci. 42 (2007), p. 1373.

[9] MG Seitz et al., Science 182 (1974), p. 954.

[10] AN Krot et al., Meteoritics 30 (1995), p. 748.

[11] We gratefully acknowledge NASA (grants \#NNX12AL47G and \#NNX15AJ22G) and NSF (grants \#1531243 and \#0619599) for funding of instrumentation in the Kuiper Materials Imaging and Characterization Facility at UA/LPL. Research supported by NASA grant \#NNX15AJ22G.

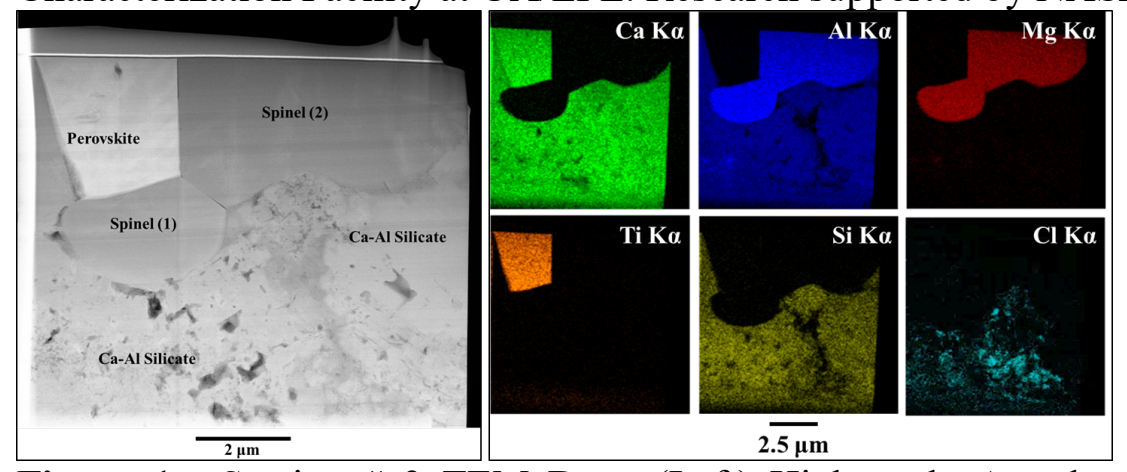

Figure 1. Section \# 3 TEM Data. (Left) High-angle Annular Dark-field (HAADF) Image; (Right) False-color EDS Maps. 